\title{
Optimal (Solvent) Mixture Design through a Decomposition Based CAMD methodology
}

\author{
Achenie, L.; Karunanithi, Arunprakash T.; Gani, Rafiqul
}

Published in:

European Symposium on Computer Aided Process Engineering - 14

Link to article, DOI:

10.1016/S1570-7946(04)80102-0

Publication date:

2004

Document Version

Early version, also known as pre-print

Link back to DTU Orbit

Citation (APA):

Achenie, L., Karunanithi, A. T., \& Gani, R. (2004). Optimal (Solvent) Mixture Design through a Decomposition Based CAMD methodology. In European Symposium on Computer Aided Process Engineering - 14 (pp. 217222). Elsevier. Computer Aided Chemical Engineering Vol. 18 https://doi.org/10.1016/S1570-7946(04)80102-0

\section{General rights}

Copyright and moral rights for the publications made accessible in the public portal are retained by the authors and/or other copyright owners and it is a condition of accessing publications that users recognise and abide by the legal requirements associated with these rights.

- Users may download and print one copy of any publication from the public portal for the purpose of private study or research.

- You may not further distribute the material or use it for any profit-making activity or commercial gain

- You may freely distribute the URL identifying the publication in the public portal 


\title{
Optimal (Solvent) Mixture Design through a Decomposition Based CAMD methodology
}

\author{
Arunprakash T.Karunanithi ${ }^{1}$ and Luke E.K.Achenie ${ }^{1 *}$, Rafiqul Gani ${ }^{2}$ \\ ${ }^{1}$ Department of Chemical Engineering, University of Connecticut, \\ Storrs, CT-06269, USA \\ ${ }^{2}$ CAPEC,Department of Chemcial Engineering, \\ Technical University of Denmark, DK-2800 Lyngby, Denmark
}

\begin{abstract}
Computer Aided Molecular/Mixture design (CAMD) is one of the most promising techniques for solvent design and selection. A decomposition based CAMD methodology has been formulated where the mixture design problem is solved as a series of molecular and mixture design sub-problems. This approach is able to overcome most of the difficulties associated with the solution of mixture design problems. The new methodology has been illustrated with the help of a case study involving the design of solvent-anti solvent binary mixtures for crystallization of Ibuprofen.
\end{abstract}

Keywords: CAMD,MINLP,Optimization, Mixture Design, Solvents, Ibuprofen

\section{Introduction}

Computer Aided Molecular/Mixture Design (CAMD) is a very promising technique for design of solvents, (solvent) mixtures and/or formulations for different processes. CAMD technique has been used previously for the design of solvents and a review of the various solution techniques can be found in Achenie et al.,(2002). The authors define computer aided molecular design as "Given a set of building blocks and a set of target properties, determine the molecule or molecular structure that matches these properties'. In this technique the reverse problem of property estimation is tackled, that is, for a specified set of properties (target properties), pure compounds or mixtures that satisfy the property requirements are determined. The CAMD problem can be posed as a mixed integer non-linear programming (MINLP) problem, where a (process-product) performance index is optimised subject to constraints such as process models, product characteristics, molecular generation rules etc. The resulting MINLP model can be solved in many ways. The most direct approach is to completely enumerate the MINLP model to give several non-linear programs (NLP) corresponding to fixed values of the integer variables. A simple generate and test approach is an example of complete enumeration. Achenie et al., however report of developments based on a multi level approach, which avoids complete enumeration. For practical solvent and mixture design

\footnotetext{
* Author to whom correspondence should be adressed : achenie@engr.uconn.edu
} 
problems, solving the CAMD MINLP model using standard optimisation approaches presents several difficulties. These arise from the need to use highly non-linear and complex property models for the prediction of the product-process characteristics, the need to relate the product function with the process model (where the process model equations are represented as a set of equality constraints), the search space, global optimal issues and many more. In this work we have developed a new CAMD methodology where the general molecule-mixture design problem (posed as an MINLP problem) is decomposed into an ordered set of sub-problems, that combined together, represent the original problem definition. The advantage is a more flexible solution approach together with easy to solve sub-problems related to various features of the molecule-mixture design problem.

\section{Methodology}

A general CAMD problem (Molecular and Mixture design) formulated as an MINLP is shown below.

$\operatorname{Min} / \operatorname{Max} f_{o b j}(X, Y)$

S.t Structural constraints: $g_{l}(Y) \leq 0$

Pure component property constraints: $g_{2}(Y) \leq 0$

Mixture property constraints: $g_{3}(X, Y) \leq 0$

Process model constraints: $g_{4}(X, Y) \leq 0$

$Y$ is a vector of integer variables, which are related to the identities of the building blocks and/or molecules. $X$ is a vector of continuous variables, which are related to the mixture (e.g., compositions) and/or process variables (e.g., flow rates, temperatures $e t c$.). $f_{o b j}$ is the objective function, which defines the optimisation objective in terms of mixture-process (performance) characteristics and/or cost that may be minimized or maximized. $g_{1}$ and $g_{2}$ are sets of structural (related to feasibility of molecular structure) constraints and pure component property (related to properties-molecular structure relationships) constraints respectively. $g_{3}$ and $g_{4}$ are set of mixture property (related to properties-mixture relationships) constraints and process model (related to processmolecule/mixture relationships) constraints respectively.

The general CAMD problem is first decomposed into two parts molecular design and mixture design. If we are interested in pure component solvent design then only the first part is followed while if we are interested in mixture design both parts are followed (see Fig. 1). In the first part (pure component design) the problem is decomposed into four sub-problems. The first sub-problem considers the structural constraints that result in generation of feasible molecular structures. The second sub-problem considers the pure component properties, and the feasible molecular structures from the first sub-problem are solved for the pure component properties. Those molecules, which satisfy the pure component property constraints, are then passed into the third sub-problem, which considers the mixture properties. Those satisfying the mixture property constraints are passed on to the final sub-problem where the process model constraints are considered along with the objective function and the optimal solvent is identified by either solving a smaller MINLP problem or a series of NLP problems or just ranking the solvents 
according to the performance index, depending on the number of feasible solutions that are passed into the final sub-problem.

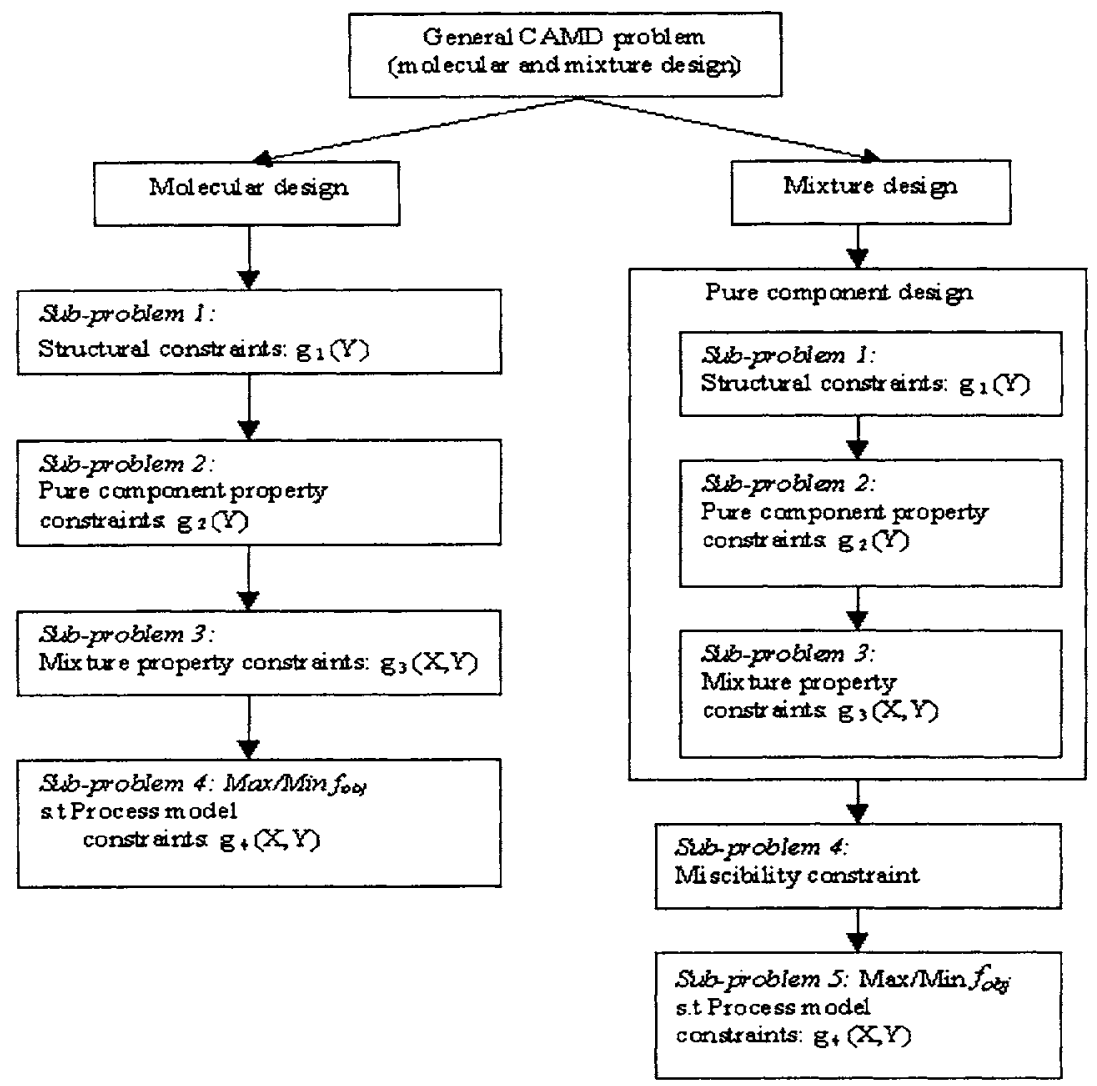

Figure 1:Flow diagram of decomposition methodology

The second part deals with mixture design. In this part promising pure component solvents are designed first and then the solvent mixture is identified. The mixture design problem is also solved as a series of sub-problems. The first three sub-problems deal with the design of pure component solvents and the final two sub-problems deal with mixture design. At the end of sub-problem 3, we will have all promising pure component solvents. In the fourth sub-problem mixture property constraints concerning the miscibility of the solvents among themselves in the mixture (e.g. miscibility of two solvents if a binary mixture is designed) is considered. In the final sub-problem the process model constraints are considered along with the objective function and the optimal mixture is identified by solving a MINLP problem or a series of NLP problems or ranking the mixtures according to the performance index. In some mixture design problems (such as formulations), it may not be necessary to consider processing issues and hence we would not have the process model constraints and the problem becomes a simple mixing problem, which would already have been addressed 
by the miscibility criteria in the sub-problem 4 . Hence, for these problems, we will not need the sub-problem 5 . Also in some cases we might have to identify a mixture whose constituents perform different jobs. In that case we would have to formulate and solve more than one pure component design problems to identify the constituents and then solve the final two sub-problems to identify the optimal mixture.

\section{Case Study: Solvent-Anti solvent design for Ibuprofen}

Ibuprofen is an important pharmaceutical compound, which is usually produced by drowning out crystallization. In this method the drug (Ibuprofen) is crystallized out of the solution as its solubility decreases and super saturation is generated when a very poor solvent called anti-solvent is added. In the simplest and most common drowning out process, the anti-solvent is miscible with the mother liquor at all process conditions (Frank et al., 1999). The solvent designed should have high solubility for Ibuprofen so that the quantity of solvent required is minimal. The choice of solvents directly influences the morphology of crystals obtained. So it is extremely important that the appropriate solvents and anti solvents re selected. Gordon and Amin (1984), say that better shaped crystals (equant shaped) are obtained when solvents having hydrogen bonding solubility parameter of atleast 8 Hilderband units are used as against aliphatic hydrocarbon solvents, which yield rod or needle shaped crystals.

\subsection{Solvent-Anti solvent property requirements}

The solvent and anti-solvent should be liquid at operating conditions. Hence constraints on boiling point $\left(T_{b}\right)$ and melting point $\left(T_{m}\right)$ are imposed on the designed solvent and anti-solvent. The solvent should have very high solubility for Ibuprofen. Usually solvents having solubility parameter $(\delta)$ value close to that of the solute have high solubility for the solute. The estimated solubility parameter of Ibuprofen is 19 $\mathrm{MPA}^{1 / 2}$. Hence the designed solvent should have solubility parameter values close to 19 $\mathrm{MPA}^{1 / 2}$.The solvent designed should be able to produce equant shaped crystals. For purposes of screening, a constraint on hydrogen bonding solubility parameter $\left(\delta_{H}\right)$ of the solvent is imposed. The designed anti solvent should have low solubility for Ibuprofen. A constraint on solubility parameter takes care of this. The solvent and anti solvent should be completely miscible in the whole composition range. The mixture should have high solubility for the solute at higher compositions of solvent and low solubility for the solute at higher compositions of anti-solvent so that the yield will be high. The various properties are estimated using group contribution methods.

\subsection{Mathematical formulation}

The overall mixture (solvent-anti solvent) design problem is shown below Max Solubility difference $f_{o b j}(X, Y)$

s.t. Structural constraints: $g_{1}(Y) \leq 0$

Pure component property constraints: $g_{2}$ (functional groups, group contributions) $\leq 0$

$T_{b}$ (solvent) $\geq 340$; Normal boiling point

$T_{m}$ (solvent) $\leq 270$; Normal melting point

$18 \leq \delta$ (solvent) $\leq 19$; Hildebrand solubility parameter

$\delta_{H}$ (solvent) $\geq 8$; Hansen (Hydrogen-bonding) solubility parameter 


\author{
$T_{b}$ (anti-solvent) $\geq 340$; Normal boiling point \\ $T_{m}$ (anti-solvent) $\leq 270$; Normal melting point \\ $\delta$ (anti-solvent) $\leq 29$; Hildebrand solubility parameter \\ Mixture property constraints: Solvent-Anti solvent miscibility \\ $g_{3}$ (compound ID, temperature, model parameters, composition) $=0$
}

Following the second part (see Fig. 1) solves this mixture design problem, as stated in the methodology (see section 2). Pure component solvents and anti-solvents are designed first and then optimal solvent-anti solvent binary mixture is identified. Since solvents and anti-solvents perform different roles and hence have different property constraints two molecular design problems need to be formulated and solved. As stated in the methodology the first three sub-problems pertain to pure component design. For the pure component solvent design, the structural constraints are considered in the first sub-problem. In the second sub-problem all feasible molecular structures were solved for the four pure component property constraints of the solvent namely boiling point, melting point, total solubility parameter and hydrogen bonding solubility parameter. Since we do not have mixture property constraints regarding pure solvents we do not need the third sub-problem in the methodology. The same applies to the three subproblems of the pure component anti-solvent design where anti-solvent property constraints namely boiling point, melting point and solubility parameter are considered. Then the fourth sub-problem as per the methodology is solved where the pure component solvents and anti solvents are verified for the binary miscibility. In this case study the process model constraints are not considered and hence we do not need the final sub-problem. Now the optimal solvent is selected by evaluating the performance index (objective function) of all the binary solvent - anti solvent mixtures that satisfied all the sub-problems. The sub problems have been solved with the ProCAMD toolbox in ICAS (ICAS Documentations, 2002).

\title{
3.3 Results
}

For the solvent design in the first sub-problem only acyclic compounds were designed. 3498 feasible molecular structures were generated in the first sub-problem. In the second sub-problem from the 3498 molecules only 55 molecular structures satisfied the four pure component property constraints of the solvent. Sub-problem 3 was bypassed as solubilities were calculated in sub-problem 2 . These generated molecules were compared with a database of compounds (to identify if they exist or has been produced as a chemical) and 7 of these were found to exist. These seven molecules were selected as potential solvent candidates. For the anti-solvent design, also 3498 feasible molecular structures were generated in the first sub-problem. In the second sub-problem only two out of the 3498 structures satisfied the three anti-solvent pure component property constraints. The third sub-problem was again omitted. These two solvents were selected as potential anti-solvents. Now in the fourth sub-problem only six pairs were found to be completely miscible with each other. As stated before we did not consider the process model in the fifth sub-problem - we only need to find the mixture that best satify the performance index. This is now simple, as we only need to evaluate the performance index for each of the identified six binary mixtures and rank them. The 
performance index is the difference in the solubility of Ibuprofen at high $(0.8$ mole percent) and low ( 0.1 mole percent) compositions of solvent. The pair that had the highest solubility difference (objective $=$ Solubility $\left(\mathrm{X}_{1}{ }^{\text {sat }}\right.$, Solvent high, anti-solvent low)- Solubility $\left(\mathrm{X}_{2}^{\text {sat }}\right.$, Solvent low, anti-solvent high)) was Propionic anhydride (Solvent)- 1,3 Propylene glycol (Anti solvent), which was therefore selected as the optimal solvent and anti-solvent mixture. Gordon and Amin (1984) state that Ibuprofen is commercially crystallized from hexane or heptane and the crystals are rod or needle shaped and argue that if the $\delta_{H}$ value of the solvent is $>8$ it is possible to obtain equant shaped crystals. Our designed binary mixture should be able to produce better crystals as compared to heptane or hexane, since the solvent has higher solubility. Note, however, that for final verification, experiments would need to be performed, which is outside the scope of this work.

Table 1: Design Results (Estimated Properties)

Solvent: Propionic anhydride

Anti solvent: 1,3 Propylene glycol

$\mathrm{T}_{\mathrm{m}}=237.34 \mathrm{~K}$

$$
\begin{aligned}
\mathrm{T}_{\mathrm{m}} & =235.53 \mathrm{~K} \\
\mathrm{~T}_{\mathrm{b}} & =453.47 \mathrm{~K} \\
\delta & =30.51 \mathrm{MPA}^{1 / 2}
\end{aligned}
$$

$\mathrm{T}_{\mathrm{b}}=450.62 \mathrm{~K}$

$\delta=19.82 \mathrm{MPA}^{\mathrm{I} / 2}$

\section{Conclusions}

This paper presents a new decomposition based methodology for solving mixture design problems. The mixture design problem formulated as an MINLP model is solved as a series of sub-problems. These sub-problems are sub-set of constraints from the original set and are easy to solve. The problem becomes smaller and simpler as each subproblem is solved. The Ibuprofen case study illustrates that the methodology can be applied to solve practical solvent design problems. It also shows that CAMD techniques can help to reduce the number of alternatives and to design the experiments needed for final verification, thereby, saving much time and resources.

\section{References}

Achenie, L.E.K., Gani, R. and Venkatsubrmaniam, V., 2002, Computer aided molecular design: theory and practice, Elsevier.

Frank, T.C., Downey, J.R. and Gupta, S.K., 1999, Quickly screen solvents for organic solids, Chem. Eng. Prog. 95(12), 41-61.

Gordon, R.E. and Amin, S.I., 1984, Crystalliztion of Ibuprofen, U.S.Patent number $4,476,248$.

ICAS Documentations, 2002, CAPEC Internal Report PEC-0214, KT, DTU, Lyngby, Denmark.

Nass, K.K., 1994, Rational solvent selection for cooling crystallizations, Ind. Eng. Chem. Res., 33, 1580-1584. 